\title{
Optimization of temperature field evolution simulation during wet flat grinding
}

\author{
J. L. González-Santander ${ }^{1}$, G. Martín ${ }^{1}$, E. Moreno Gálvez ${ }^{2}$ \\ \& D. Yáñez ${ }^{2}$ \\ ${ }^{I}$ Dpto. Ciencias Experimentales y Matemáticas, Universidad Católica de \\ Universidad Católica de Valencia "san Vicente mártir", Spain \\ ${ }^{2}$ Dpto. Matemáticas, CC. NN. y CC. SS. aplicadas a la educación, \\ Universidad Católica de Valencia "san Vicente mártir", Spain
}

\begin{abstract}
We have considered the analytical solutions for heat transfer in wet flat grinding, assuming a linear and a constant heat flux profile, entering into the workpiece. We assume as well a constant heat transfer coefficient for the coolant acting on the workpiece surface. In order to avoid thermal damage, we provide a very fast method for the computation of the maximum temperature, which occurs on the workpiece surface in the stationary regime. We also provide a very rapid method for the numerical evaluation of the transient regime duration (relaxation time). By knowing the location of the maximum temperature and the relaxation time, we have performed an analysis for the computation optimization of the temperature evolution on the workpiece surface. This kind of analysis offers a very interesting simulation tool to avoid thermal damage during the transient regime.

Keywords: flat grinding, wet grinding, thermal damage, surface temperature in grinding.
\end{abstract}

\section{Introduction}

Flat grinding is an industrial machining process consisting in removing the surface roughness of a workpiece by means of an abrasive wheel which rotates at high speed sliding over its surface (see Figure 1). Most of the energy used in the grinding process is converted into heat, and it is accumulated within the contact zone between the wheel and the workpiece [1]. The high temperatures reached can damage the quality of the workpiece, producing residual stresses and even 


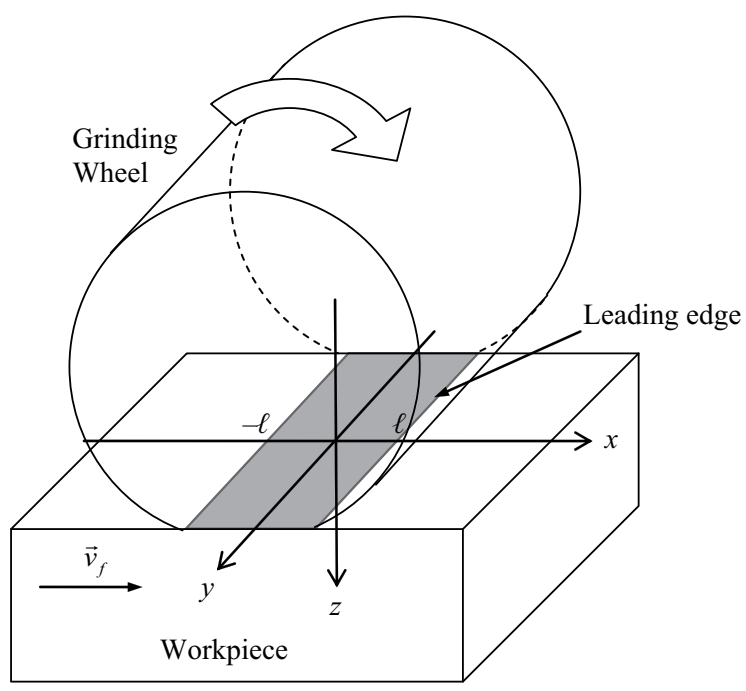

Figure 1: Strip heat source on a semi-infinite workpiece due to friction with a grinding wheel.

burning the surface layers of the workpiece [2]. Therefore, the determination of the temperature field evolution inside the workpiece is of great industrial importance [3-6]. In order to avoid thermal damage, liquid coolant is usually injected onto the workpiece surface (wet grinding), so power generation by friction is reduced and cooling by convection occurs.

\section{Analytical approach}

In flat grinding, the heat transfer inside the workpiece usually is modeled by a strip heat source infinitely long and of $2 \ell$ width (m, SI units), which moves at a speed $\vec{v}_{f}=v_{f} \vec{i}\left(\mathrm{~ms}^{-1}\right)$ over a semi-infinite solid surface (see Figure 1). Taking a coordinate system $(x, y, z)$ fixed to the heat source, the workpiece temperature field $T(x, z, t)$ satisfies the convective heat equation $[7, \S 1.7(2)]$

$$
\frac{\partial T}{\partial t}=k\left(\frac{\partial^{2} T}{\partial x^{2}}+\frac{\partial^{2} T}{\partial z^{2}}\right)-v_{f} \frac{\partial T}{\partial x},
$$

where $k$ is the thermal diffusivity $\left(\mathrm{m}^{2} \mathrm{~s}^{-1}\right)$. The convective heat equation (1) is subjected to the initial condition

$$
T(0, x, z)=T_{0},
$$

where $T_{0}$ is the room temperature.

Wet grinding can be modelled assuming a constant heat transfer coefficient $h$ $\left(\mathrm{W} \mathrm{m} \mathrm{m}^{-2} \mathrm{~K}^{-1}\right.$ ) on the workpiece surface. Assuming as well a dimensionless heat 
flux profile $f(x)$ within the contact area between wheel and workpiece, we have the following boundary condition

$$
k_{0} \frac{\partial T}{\partial z}(t, x, 0)=h\left[T(x, 0, t)-T_{0}\right]-q f(x) \mathcal{H}(x-\ell) \mathcal{H}(x+\ell)
$$

where $\mathcal{H}(x)$ is the Heaviside function, $k_{0}$ is the thermal conductivity and $q$ (W $\mathrm{m}^{-2}$ ) is a constant that takes account the amount of heat flux entering into the workpiece.

In order to solve the problem given in (1)-(3) we can build the solution departing from the Green function $G\left(\vec{r}, t ; \vec{r}^{\prime}, t^{\prime}\right)$, which in this case is interpreted as the temperature field evaluated at $(\vec{r}, t)=(x, y, z, t)$ in a semi-infinite body $(z>0)$ in which at position $\vec{r}^{\prime}=\left(x^{\prime}, y^{\prime}, z^{\prime}\right)$ an instantaneous point heat source of strength $Q\left(\mathrm{~m}^{3} \mathrm{~K}\right)$ appears at the instant $t^{\prime}<t$, and where the surface $(z=0)$ is subject to a constant heat transfer coefficient $h[7, \S 14.9$ (4)]

$$
\begin{aligned}
& G\left(\vec{r}, t ; \vec{r}^{\prime}, t^{\prime}\right)=\frac{Q}{4 \pi k\left(t-t^{\prime}\right)} \exp \left(-\frac{\left(x-x^{\prime}\right)^{2}+\left(y-y^{\prime}\right)^{2}}{4 k\left(t-t^{\prime}\right)}\right) \\
& \times\left\{\frac{\exp \left(-\frac{\left(z-z^{\prime}\right)^{2}}{4 k\left(t-t^{\prime}\right)}\right)+\exp \left(-\frac{\left(z+z^{\prime}\right)^{2}}{4 k\left(t-t^{\prime}\right)}\right)}{2 \sqrt{\pi k\left(t-t^{\prime}\right)}}\right. \\
& -\frac{h}{k_{0}} \exp \left[\frac{h}{k_{0}}\left(z+z^{\prime}\right)+\frac{h^{2}}{k_{0}^{2}} k\left(t-t^{\prime}\right)\right] \\
& \left.\times \operatorname{erfc}\left(\frac{h}{k_{0}} \sqrt{k\left(t-t^{\prime}\right)}+\frac{z+z^{\prime}}{2 \sqrt{k\left(t-t^{\prime}\right)}}\right)\right\} .
\end{aligned}
$$

A formal derivation of (4) is given in [8, Appendix].

The derivation of the solution by using (4) follows three steps:

- Superposition in space to give temperature due to instantaneous line source, which acts on the surface $z^{\prime}=0$ parallel to the $y$-axis.

- Superposition in time to give temperature due to continuously-acting line source.

- Superposition of line sources to give temperature due to a continuously acting band source on the surface.

Following these steps and taking a constant heat flux profile in (3), that is

$$
f(x)=1,
$$

the solution of (1)-(3) in dimensionless variables is [8, Eqn. 8]

$$
\begin{aligned}
\mathcal{T}^{(c)} & (\tau, X, Z) \\
= & \sqrt{\pi} \int_{0}^{\tau}\left[\exp \left(\frac{-Z^{2}}{4 u^{2}}\right)-\sqrt{\pi} H u e^{H Z+H^{2} u^{2}} \operatorname{erfc}\left(H u+\frac{Z}{2 u}\right)\right] \\
& \times\left[\operatorname{erf}\left(\frac{X+L}{2 u}+u\right)-\operatorname{erf}\left(\frac{X-L}{2 u}+u\right)\right] d u
\end{aligned}
$$


where the superscript ${ }^{(c)}$ denotes we are considering a constant heat flux profile, and where we have considered the following dimensionless variables:

$$
\begin{aligned}
& X=\frac{v_{f} x}{2 k}, \quad Z=\frac{v_{f} z}{2 k}, \quad L=\frac{v_{f} \ell}{2 k}, \quad H=\frac{2 k h}{v_{f} k_{0}}, \\
& \tau=\frac{v_{f} \sqrt{t}}{2 \sqrt{k}}, \quad \mathcal{T}=\frac{\pi k_{0} v_{f}}{2 q k}\left(T-T_{0}\right) .
\end{aligned}
$$

Similarly, for a linear heat flux profile

$$
f(x)=1+\frac{x}{\ell}
$$

the solution of (1)-(3) in dimensionless variables is

$$
\begin{aligned}
\mathcal{T}^{(l)}( & \tau, X, Z) \\
= & \frac{1}{L} \int_{0}^{\tau}\left[\exp \left(\frac{-Z^{2}}{4 u^{2}}\right)-\sqrt{\pi} H u e^{H Z+H^{2} u^{2}} \operatorname{erfc}\left(H u+\frac{Z}{2 u}\right)\right] \\
& \times\left\{\sqrt{\pi}\left(X+L+2 u^{2}\right)\left[\operatorname{erf}\left(\frac{X+L}{2 u}+u\right)-\operatorname{erf}\left(\frac{X-L}{2 u}+u\right)\right]\right. \\
& \left.+2 u\left[\exp \left(-\left(\frac{X+L}{2 u}+u\right)^{2}\right)-\exp \left(-\left(\frac{X-L}{2 u}+u\right)^{2}\right)\right]\right\} d u
\end{aligned}
$$

where the superscript ${ }^{(l)}$ indicates we are considering a linear heat flux profile. Performing the change of variables $w=2 u^{2}$ and the limit $\tau \rightarrow \infty$, we obtain the following expression for the stationary regime, which is given in [9, Eqns. 3.4647],

$$
\begin{aligned}
\mathcal{T}_{\text {wet }}^{(l)} & (X, Z) \\
= & \lim _{\tau \rightarrow \infty} \mathcal{T}_{\text {wet }}^{(l)}(\tau, X, Z) \\
= & \frac{1}{2 L} \int_{0}^{\infty} \exp \left(\frac{-Z^{2}}{2 w}\right) \\
& \times\left\{\sqrt{\frac{\pi}{2 w}}(X+L+w)\left[\operatorname{erfc}\left(\frac{X+L+w}{\sqrt{2 w}}\right)-\operatorname{erfc}\left(\frac{X-L+w}{\sqrt{2 w}}\right)\right]\right. \\
& \left.+\exp \left(-\frac{(X+L+w)^{2}}{2 w}\right)-\exp \left(-\frac{(X-L+w)^{2}}{2 w}\right)\right\} d w \\
& -\frac{\pi H}{4 L} \int_{0}^{\infty} \exp \left(\frac{-Z^{2}}{2 w}\right) \exp \left(\frac{H^{2} w}{2}\right) \operatorname{erfc}\left(\frac{H w}{\sqrt{2 w}}\right)
\end{aligned}
$$




$$
\begin{aligned}
& \times\left\{(X+L+w)\left[\operatorname{erfc}\left(\frac{X-L+w}{\sqrt{2 w}}\right)-\operatorname{erfc}\left(\frac{X+L+w}{\sqrt{2 w}}\right)\right]\right. \\
& \left.+\sqrt{\frac{2 w}{\pi}}\left[\exp \left(-\frac{(X+L+w)^{2}}{2 w}\right)-\exp \left(-\frac{(X-L+w)^{2}}{2 w}\right)\right]\right\} d w .
\end{aligned}
$$

From a computational point of view, the infinite upper limits of (8) and (9) can be replaced by [9, Eqn. 3.60]

$$
w_{\max }=2 L-X+\frac{Z}{2}+10
$$

which, according to the change of variables performed, provides a simple formula for the duration of the transient regime (relaxation time)

$$
\tau_{\max }(X, Z)=\sqrt{L-\frac{X}{2}+\frac{Z}{4}+5}
$$

However, equation (10) is a fitting formula that is not obtained from any analytical consideration. In Section 4 we will derive some expressions for the relaxations times and in Section 5 we will compare them to (10) numerically.

\section{Maximum temperature}

From a physical point of view, the maximum temperature $\mathcal{T}_{\text {max }}$ must be reached in the stationary regime and on the workpiece surface, $Z=0$, just within the contact zone between the grinding wheel and the workpiece $X_{\max } \in(-L, L)$. Therefore, we have to search the local extrema of $\mathcal{T}(X, 0)$ for $X \in(-L, L)$, that is

$$
\frac{\partial \mathcal{T}(X, 0)}{\partial X}=0, \quad X \in(-L, L)
$$

In order to obtain the maximum temperature, from all the local extrema $X_{k}$ found in (11), we will choose the global maximum, that is

$$
\mathcal{T}_{\max }=\mathcal{T}\left(X_{\max }, 0\right)=\max _{k}\left(\mathcal{T}\left(X_{k}, 0\right)\right)
$$

For a constant heat flux profile, according to (5), we have

$$
\begin{aligned}
\mathcal{T}^{(c)} & (X, 0) \\
= & \sqrt{\pi} \int_{0}^{\infty}\left[1-\sqrt{\pi} H u e^{H^{2} u^{2}} \operatorname{erfc}(H u)\right] \\
& \times\left[\operatorname{erf}\left(\frac{X+L}{2 u}+u\right)-\operatorname{erf}\left(\frac{X-L}{2 u}+u\right)\right] d u .
\end{aligned}
$$


According to (11) and (12), we have to evaluate numerically within the interval $X \in(-L, L)$ the following equation:

$$
\begin{aligned}
0= & \int_{0}^{\infty}\left[\frac{1}{u}-\sqrt{\pi} H e^{H^{2} u^{2}} \operatorname{erfc}(H u)\right] \\
& \times\left\{\exp \left(-\left[\frac{X+L}{2 u}+u\right]^{2}\right)-\exp \left(-\left[\frac{X-L}{2 u}+u\right]^{2}\right)\right\} d u .
\end{aligned}
$$

Similarly, from (6), we can get the stationary regime on the workpiece surface for a linear heat flux profile, obtaining

$$
\begin{aligned}
\mathcal{T}^{(l)} & (X, 0) \\
= & \frac{1}{L} \int_{0}^{\infty}\left[1-\sqrt{\pi} H u e^{H^{2} u^{2}} \operatorname{erfc}(H u)\right] \\
& \times\left\{\sqrt{\pi}\left(X+L+2 u^{2}\right)\left[\operatorname{erf}\left(\frac{X+L}{2 u}+u\right)-\operatorname{erf}\left(\frac{X-L}{2 u}+u\right)\right]\right. \\
& \left.-2 u\left[\exp \left(-\left[\frac{X+L}{2 u}+u\right]^{2}\right)-\exp \left(-\left[\frac{X-L}{2 u}+u\right]^{2}\right)\right]\right\} d u
\end{aligned}
$$

and the equation we have to solve numerically within the interval $X \in(-L, L)$ in order to get the maximum temperature is

$$
\begin{aligned}
0= & \int_{0}^{\infty}\left[1-\sqrt{\pi} H u e^{H^{2} u^{2}} \operatorname{erfc}(H u)\right] \\
& \times\left\{\sqrt{\pi}\left[\operatorname{erf}\left(\frac{X+L}{2 u}+u\right)-\operatorname{erf}\left(\frac{X-L}{2 u}+u\right)\right]\right. \\
& \left.-\frac{2 L}{u} \exp \left(-\left[\frac{X-L}{2 u}+u\right]^{2}\right)\right\} d u .
\end{aligned}
$$

\section{Relaxation time}

The stationary regime is asymptotically reached at $t \rightarrow \infty$, i.e.

$$
\lim _{\tau \rightarrow \infty} \frac{\partial \mathcal{T}(\tau, X, Z)}{\partial \tau}=0
$$

In order to avoid thermal damage, the most important point is the location of the maximum temperature. Therefore, for estimating how rapidly the stationary regime is reached (i.e. the relaxation time of the transient regime), we can solve 
the following equation for $\bar{\tau} \in(0, \infty)$ :

$$
\left.\frac{\partial \mathcal{T}(\bar{\tau}, X, 0)}{\partial \tau}\right|_{X=X_{\max }}=\eta \approx 0
$$

For a constant heat flux profile, according to (5), equation (17) is written as

$$
\begin{aligned}
\eta= & \left.\sqrt{\pi}\left[\operatorname{erf}\left(\frac{X+L}{2 \bar{\tau}}+\bar{\tau}\right)-\operatorname{erf}\left(\frac{X-L}{2 \bar{\tau}}+\bar{\tau}\right)\right]\right|_{X=X_{\max }} \\
& \times\left[1-\sqrt{\pi} H \bar{\tau} e^{H^{2} \bar{\tau}^{2}} \operatorname{erfc}(H \bar{\tau})\right]
\end{aligned}
$$

Let us solve (18) approximately. On the one hand, according to the asymptotic expansion given in the literature [10, Eqn. 7.1.23] for the erfc function, we have

$$
1-\sqrt{\pi} H \bar{\tau} e^{H^{2} \bar{\tau}^{2}} \operatorname{erfc}(H \bar{\tau}) \approx \frac{1}{2 H^{2} \bar{\tau}^{2}}, \quad \bar{\tau} \rightarrow \infty .
$$

In the other hand, expanding the Taylor series of the error function up to the first order, we have

$$
\sqrt{\pi}\left[\operatorname{erf}\left(\frac{X+L}{2 \bar{\tau}}+\bar{\tau}\right)-\operatorname{erf}\left(\frac{X-L}{2 \bar{\tau}}+\bar{\tau}\right)\right] \approx \frac{2 L}{\bar{\tau}} e^{-\bar{\tau}^{2}}, \quad \bar{\tau} \rightarrow \infty
$$

Substituting (19) and (20) in (18), we get the following approximated equation

$$
\bar{\tau}^{3} e^{\bar{\tau}^{2}} \approx \frac{L}{H^{2} \eta}
$$

By using the Lambert W function [11], (21) can be solved explicitly, obtaining the following approximated expression for the relaxation time, considering a constant heat flux profile in the wet case

$$
\bar{\tau}^{(c)} \approx \sqrt{\frac{3}{2} \mathrm{~W}\left(\frac{2}{3}\left[\frac{L}{H^{2} \eta}\right]^{2 / 3}\right)} .
$$

The approximation given in (22) can be used as the starting iteration point for the numerical evaluation of (18). Notice that (22) do not depend on $X_{\max }$, so we do not have to compute (13) if we want an estimation of the relaxation time for a constant heat flux profile. 
Considering now a linear heat flux profile, equation (17) is written as

$$
\begin{aligned}
\eta= & \frac{1}{L}\left[1-\sqrt{\pi} H \bar{\tau} e^{H^{2} \bar{\tau}^{2}} \operatorname{erfc}(H \bar{\tau})\right] \\
& \times\left\{\sqrt{\pi}\left(X+L+2 \bar{\tau}^{2}\right)\left[\operatorname{erf}\left(\frac{X+L}{2 \bar{\tau}}+\bar{\tau}\right)-\operatorname{erf}\left(\frac{X-L}{2 \bar{\tau}}+\bar{\tau}\right)\right]\right. \\
& \left.-2 \bar{\tau}\left[\exp \left(-\left[\frac{X+L}{2 \bar{\tau}}+\bar{\tau}\right]^{2}\right)-\exp \left(-\left[\frac{X-L}{2 \bar{\tau}}+\bar{\tau}\right]^{2}\right)\right]\right\}\left.\right|_{X=X_{\max }} .
\end{aligned}
$$

In order to solve (23) approximately, we can expand the Taylor series of the $e^{-x^{2}}$ function up to first order, so we can write

$$
\exp \left(-\left[\frac{X+L}{2 \bar{\tau}}+\bar{\tau}\right]^{2}\right)-\exp \left(-\left[\frac{X-L}{2 \bar{\tau}}+\bar{\tau}\right]^{2}\right) \approx 2 L e^{-\bar{\tau}^{2}}, \quad \bar{\tau} \rightarrow \infty
$$

Now, substituting (19), (20) and (24) in (23), we arrive at

$$
\left.\bar{\tau}^{3} e^{\bar{\tau}^{2}} \approx \frac{X+L}{H^{2} \eta}\right|_{X=X_{\max }}
$$

that can be solved explicitly as

$$
\left.\bar{\tau}^{(l)} \approx \sqrt{\frac{3}{2} \mathrm{~W}\left(\frac{2}{3}\left[\frac{X+L}{H^{2} \eta}\right]^{2 / 3}\right)}\right|_{X=X_{\max }} .
$$

As aforementioned, (25) can be used as the starting iteration point for the numerical evaluation of (23). Notice that the relaxation time $\bar{\tau}$ can be defined for each point $X$ on the workpiece surface and depends on the tolerance parameter $\eta$

$$
\bar{\tau}=\bar{\tau}(X, \eta)
$$

The computation of the relaxation time is quite useful for the numerical evaluation of the surface temperature in the stationary regime $\mathcal{T}(X, 0)$, because, according to (17), the temperature field hardly evolves over time when $\eta \ll 1$, so

$$
\mathcal{T}(X, 0) \approx \mathcal{T}(\bar{\tau}(X, \eta), X, 0), \quad \eta \ll 1
$$

Notice that the numerical evaluation of the right hand of (26) side is simpler because involves a definite integral, instead of the improper integral of the left hand side. 


\section{Numerical results}

For the numerical simulation, we have used a VT20 titanium alloy [12] as workpiece, whose thermal properties values are:

$$
k_{0}=13 \mathrm{~W} \mathrm{~m}^{-1} \mathrm{~K}^{-1}, \quad k=4.23 \times 10^{-6} \mathrm{~m}^{2} \mathrm{~s} .
$$

Also, we have used the following values for the grinding regime:

$$
\begin{aligned}
v_{f} & =0.53 \mathrm{~m} \mathrm{~s}^{-1}, \quad 2 \ell=2.663 \times 10^{-3} \mathrm{~m}, \quad q=5.89 \times 10^{7} \mathrm{~W} \mathrm{~m}^{-2}, \\
h & =2.729 \times 10^{5} \mathrm{~W} \mathrm{~m}^{-2} \mathrm{~K}^{-1}, \quad T_{0}=300 \mathrm{~K} .
\end{aligned}
$$

The following Table shows the maximum temperature rise $\Delta T_{\max }$ with respect to room temperature $T_{0}$ and its location within the friction zone, calculated according to the equations given in Section 3.

\begin{tabular}{|c||c|c|}
\hline Friction profile & $x_{\max } / \ell$ & $\Delta T_{\max }[\mathrm{K}]$ \\
\hline \hline Constant & -0.973 & 177.6 \\
\hline Linear & 0.690 & 237.3 \\
\hline
\end{tabular}

The following Table shows the relaxation times calculated according to the approximated formulae and the exact values given in Section 4, taking as tolerance parameter $\eta=10^{-3}$. Also, the relaxation time given by Sauer in (10) for $X=X_{\max }$ and $Z=0$ is provided. Notice that the initial guess given in the first column of the Table differs less than one order of magnitude with respect to the exact value.

\begin{tabular}{|c||c|c|c|}
\hline Friction profile & $\bar{t}[\mathrm{~ms}]$ (approx.) & $\bar{t}[\mathrm{~ms}]$ (exact) & $\bar{t}[\mathrm{~ms}]$ (Sauer) \\
\hline \hline Constant & 1.539 & 5.998 & 7.769 \\
\hline Linear & 1.600 & 1.658 & 3.795 \\
\hline
\end{tabular}

Figure 2 shows the temperature time evolution on the workpiece surface, considering a constant heat flux profile. For the transient regime $(t<\infty)$ we have computed (5), setting $Z=0$. For the stationary regime $(t \rightarrow \infty)$ we have computed the approximation given in (27). The evolution is so rapid that we have taken the different graphs exponentially spaced over time. Notice that the plot of the stationary regime, $t \rightarrow \infty$, is overlapped with the two last graphs, which corresponds to the values of the relaxations times (exact and Sauer) given in the previous Table. 


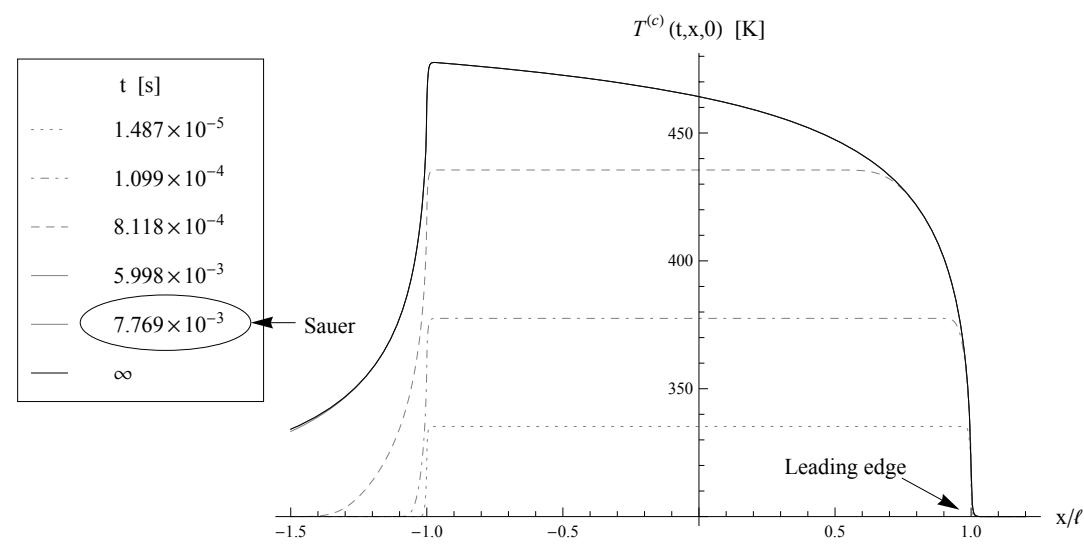

Figure 2: Time evolution of the surface temperature in wet grinding for a constant heat flux profile.

Figure 3 shows the temperature time evolution on the workpiece surface, considering a linear heat flux profile. For the transient regime $(t<\infty)$ we have computed (6), setting $Z=0$. For the stationary regime $(t \rightarrow \infty)$ we have computed the approximation given in (27). Notice that now the graphs corresponding to the relaxations times do not overlap with the stationary regime at the trailing edge. This is because the relaxation time depends on the position $X$ and for a linear heat flux profile, the maximum temperature is located far from the trailing edge, $X_{\max }>0$, just the opposite than for a constant heat flux profile, where $X_{\max } \approx-L$.

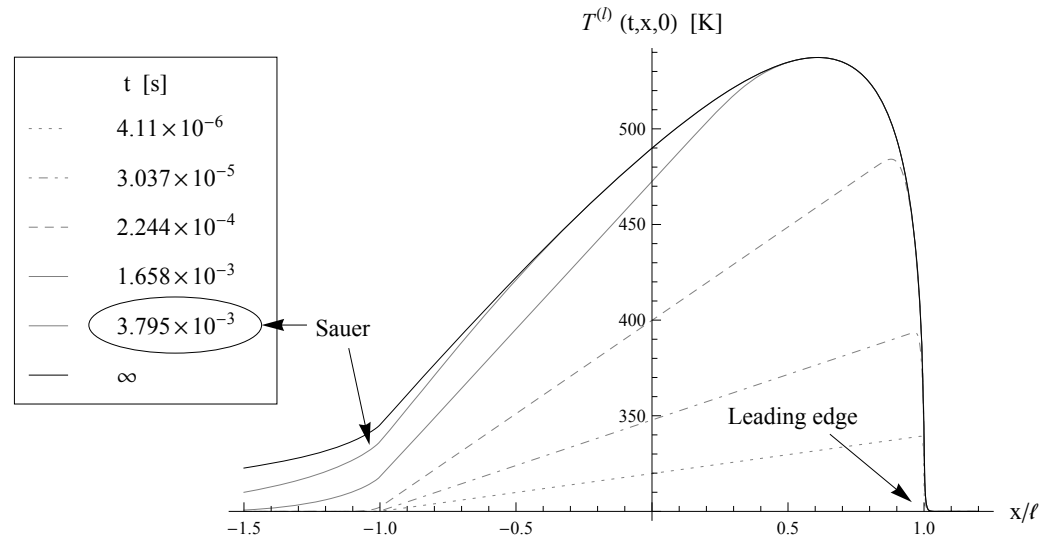

Figure 3: Time evolution of the surface temperature in wet grinding for a linear heat flux profile. 
Figure 4 shows the relaxation time as a function of the position within the contact zone for different values of the tolerance parameter $\eta$. It has been plotted only the case of a linear heat flux profile, equation (4) for $X \in(-L, L)$. It is worth noting that the numerical root finding of $(23)$ is very fast $(\approx 1 \mathrm{~s}$ for the computation of Figure 4). The behavior of the relaxation time is qualitatively similar for the constant case, as well as the computation time. As it can be seen, the relaxation time is a decreasing function from the trailing edge to the leading edge. This is so, because of the motion of the heat source. Also, the relaxation time given by Sauer is overestimated, because a naked eye can not see any difference in the temperature graphs below an error of one percent, i.e. $\eta<10^{-2}$.

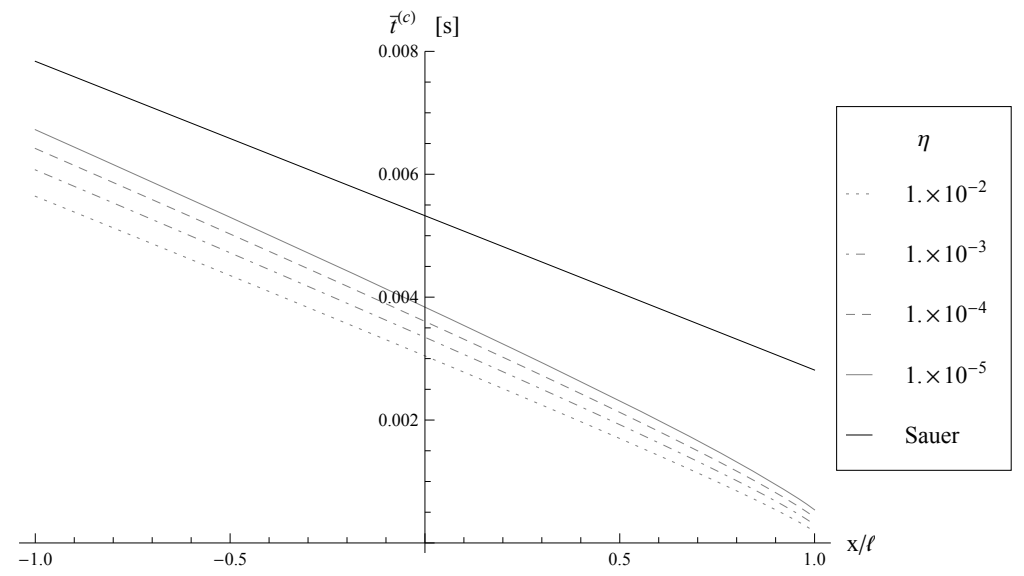

Figure 4: Relaxation time as a function of the position within the friction zone.

Since the greater relaxation time is on the trailing edge, $X=-L$, according to (26), the temperature in the stationary regime for the wet case can be approximated as

$$
\mathcal{T}(X, 0) \approx \mathcal{T}(\bar{\tau}(-L, \eta), X, 0), \quad \eta=10^{-3} .
$$

By using the above approximation, we get around a $10 \%$ reduction in the computation time for both heat flux profiles considered, i.e. constant and linear.

\section{Conclusions}

In Section 2, we have considered the analytical solutions of the time-dependant temperature field inside the workpiece in wet grinding, both with a linear heat flux profile as with a constant one within the contact zone between the wheel and the workpiece. We can find these solutions in the literature, except for the case of the transient regime considering a linear heat flux profile, i.e. (6).

Also, in Section 3, we have provided a set of equations for searching the maximum temperature in the different cases considered. It turns out that the 
root finding in these equations provides a very rapid method for the numerical evaluation of the maximum temperature. According to authors' knowledge, the equation for the linear case (15) is not given in the literature.

In order to know how rapid the stationary regime is reached, we have proposed in Section 4 a method to calculate the relaxation times for the cases considered. It turns out that the root finding that involves this method is computed very rapidly. We have compared numerically the proposed relaxation times with the fitting formula given by Sauer (10), and we have concluded that the latter overestimates them.

In order to speed up the computation of the temperature field on the workpiece surface in the stationary regime, we have used the approximation given in (27), for which we need to compute the relaxation time at the trailing edge of the heat source. Moreover, instead of computing the expression given by Sauer (7) for a linear heat flux profile, we have used (6), because involves only one integration.

\section{References}

[1] S. Malkin \& C. Guo, Grinding technology: theory and application of machining with abrasives, Industrial Press, Inc: New York, 2008.

[2] J. B. J. W Hegeman, J. Th. M. De Hosson \& G. de With, Grinding of WC-Co hardmetals, Wear, 248, pp. 187-196, 2001.

[3] C. Guo \& S. Malkin, Analysis of energy partition in grinding, J. of Eng. for Ind., 117, pp. 55-61, 1995.

[4] A. Lavine, Thermal aspects of grinding: the effects of heat generation at the shear planes, Annals of CIRP, 40, pp. 343-345, 1991.

[5] J. Pérez, S. Hoyas, D. Skuratov, Y. Ratis, I. Selezneva, P. Fernández de Córdoba \& J. Urchueguía, Heat transfer analysis of intermittent grinding processes, Int. J. Heat Mass Transfer, 51, pp. 4132-4138, 2008.

[6] C. Guo \& S. Malkin, Analysis of transient temperature in grinding, J. Manuf. Sci. Eng., 117, pp. 571-577, 1995.

[7] H. Carslaw \& J. C. Jaeger, Conduction of heat in solids, Oxford Science Publications: Oxford, 1986.

[8] J. L. González-Santander, J. M. Isidro \& G. Martín, An analysis of the transient regime temperature field in wet grinding, Journal of Engineering Mathematics, 2014, DOI: 10.1007/s10665-014-9713-6.

[9] W. J. Sauer, Thermal aspects of grinding, PhD. dissertation, Carnegie-Mellon University, 1971.

[10] M. Abramowitz \& I. Stegun, Handbook of Mathematical Functions, National Bureau of Standards: Washington, 1972.

[11] R. M. Corless, G. H. Gonnet, D. E. G. Hare, D. J. Jeffrey \& D. E. Knuth, On the Lambert W function, Advances in Computational Mathematics, 5, pp. 329-359, 1996.

[12] V. I. Murav'ev, A. V. Yakimov \& A. V. Chernysev, Effect of deformation, welding, and electrocontact heating on the properties of titanium alloy VT20 in pressed and welded structures, Metal Sci. Heat Treat., 45, pp. 419-422, 2003. 